\title{
Non-Hodgkin Lymphoma after Treatment with Extended Dosing Temozolomide and Radiotherapy for a Glioblastoma: A Case Report
}

\author{
Laura Van Ginderachter ${ }^{a}$ Tony Cox ${ }^{b}$ Ria Drijkoningen ${ }^{c}$ Ruth Achten ${ }^{c}$ \\ Eric Joosens ${ }^{a} \quad$ Annelies Maes $^{d}$ Koen Theunissen ${ }^{e}$ Jeroen Mebis ${ }^{a, f}$ \\ Departments of a Medical Oncology, ${ }^{b}$ Otorhinolaryngology, ${ }^{\mathrm{c}}$ Pathology, ${ }^{\mathrm{d}}$ Radiation \\ Oncology, and ${ }^{\mathrm{e}} \mathrm{Hematology}$, Jessa Hospital, Hasselt, and ${ }^{\mathrm{f}}$ Faculty of Medicine, \\ University of Hasselt, Diepenbeek, Belgium
}

\section{Key Words}

Temozolomide $\cdot$ B-cell lymphoma $\cdot$ Diffuse large B-cell lymphoma $\cdot$ Lymphopenia $\cdot$ Epstein-Barr virus

\begin{abstract}
Temozolomide (TMZ) is an alkylating agent, used for the treatment of high-grade gliomas. This case report describes the development of a non-Hodgkin lymphoma in a patient treated with extended-dose temozolomide and radiotherapy. In addition to the possible mutagenic effect of temozolomide - as described for all alkylating agents - there might have been an immunosuppressive effect of TMZ. The pathological appearance of the lymphoma as well as the presence of a grade 3 lymphopenia early in treatment supports this hypothesis. As the use of TMZ increases, the awareness that TMZ may induce secondary malignancies should increase as well.
\end{abstract}

\section{Introduction}

Temozolomide (TMZ) is an oral DNA alkylating agent used to treat high-grade gliomas such as glioblastoma multiforme and anaplastic astrocytoma. It results in a depletion of the DNA repair enzyme $0^{6}$-methylguanine-DNA methyltransferase (MGMT). As it improves median survival by 2.5 months and 2 -year survival rates by $16 \%$ compared to radiotherapy alone in glioblastoma patients after surgery, it has become part of the standard care of brain cancer [1]. 
The usual regimen consists of a concomitant phase and an adjuvant phase. Daily dosage of $75 \mathrm{mg} / \mathrm{m}^{2}$ for up to 6 weeks is given concomitantly with radiation therapy. In adjuvant therapy, a daily dose of $150-200 \mathrm{mg} / \mathrm{m}^{2}$ of body surface area is given for 5 days of every 28day cycle.

Alternatively, an extended dose-dense regimen, in which the dose density over the 28day cycle is $210 \%$ higher, can be administered [2]. Such regimens are believed to be more efficient as they deplete the MGMT enzyme more profoundly, but to date no significant benefit in survival has been demonstrated [2].

Temozolomide has less myelotoxicity than the traditional chemotherapy. The incidence of grade $3-4$ hematological events is believed to be less than $10 \%[3,4]$. However, as TMZ is a relatively new agent that is administered to patients with poor prognosis, possible hematological adverse events may not have been fully characterized yet.

\section{Case Report}

A 58-year-old male was admitted to the emergency department in May 2010 with a right motor hemiparesis and a speech disorder. Medical history was unremarkable except for a vasectomy and nephrolithiasis. He did not take any medication. Familial history was negative. An MRI scan showed a contrast-enhancing nodule in the left crus posterior capsulae internae of the brain. A biopsy was taken and histopathology confirmed the diagnosis of a grade IV glioblastoma. The tumor was considered inoperable by the neurosurgeon. The patient received chemoradiation with external beam radiotherapy (60 Gray in 30 fractions) and concomitant TMZ (75 mg/m²/day). Adjuvant TMZ was started in September 2010 , using a standard regimen $\left(150 \mathrm{mg} / \mathrm{m}^{2} /\right.$ day for 5 days of a 28-day cycle, or $300 \mathrm{mg}$ per cycle). Two days after the first administration, he developed epilepsy which was treated with levetiracetam (Keppra ${ }^{\odot}$ ).

MRI evaluation after 2 cycles showed tumor growth. It was then decided to start with an extended dosing schedule $\left(100 \mathrm{mg} / \mathrm{m}^{2} /\right.$ day for 21 consecutive days every 28-day cycle). From November 2010 until December 2011, 14 cycles of extended dosing TMZ were completed. Repeated imaging by MRI consistently showed good tumor control. On clinical evaluation, there was a stable dysarthria, progressive loss of memory and a tolerable headache. There was very little delay in administration of subsequent cycles, indicating low overall myelotoxicity, although a marked lymphopenia (grade 3 and sometimes grade 4) was present very early in treatment $(11 / 2010)$.

The administration of a 15th cycle, scheduled for January 2012, was delayed because of leuko- and neutropenia (grade 1). On consultation 1 month later, persisting neutropenia was seen. The patient then complained of a possible infection of the left eye, for which local oxytetracycline hydrochloride (Terramycin ${ }^{\circledR}$ ) was prescribed. One week later, he presented to the emergency unit with fever, green rhinorrhea, headache and an infection of the left eye. Neutropenia was no longer present. On chest X-ray, pneumonia was diagnosed and antibiotics were started (amoxicillin/clavulanic acid together with Trimethoprim/sulfamethoxazole). As the patient did not improve, a CT scan was performed and showed pansinusitis with an abscess breaking through the transethmoidal sinus and causing proptosis (fig. 1). Conservative treatment failed and surgical drainage was undertaken. The tissue looked very different than what could be expected from an inflammatory process. Surprisingly, the histological diagnosis was a second tumor, a high-grade B-cell lymphoma, as seen in immunodeficient patients (fig. 2). Further testing for hepatitis and HIV was 
negative. Cytomegalovirus and Epstein-Barr virus (EBV) serology was positive, consistent with an old infection.

PET-CT scanning showed involvement extending from the left ethmoid and maxillary sinuses to the naso- and oropharynx. Bone marrow investigation showed dysplasia in all 3 lineages, due to TMZ toxicity; there were no signs of invasion. Systemic therapy [rituximab, cyclophosphamide, doxorubicin, vincristine and prednisone (R-CHOP) at 50\%] was proposed; 6-8 cycles, depending on tolerance and response.

In the meantime, there was a further progression of the glioblastoma for which the patient underwent stereotactic radiosurgery of 2 tumoral localizations in the brain. The first administration of R-CHOP was not well tolerated and the patient developed disseminated zona. He and his family did not wish to proceed with therapy. Comfort care was ensured and the patient died 1 month later.

\section{Discussion}

Although alkylating agents have been associated with an increased risk of secondary neoplasms (both hematological malignancies and solid tumors), TMZ was thought to have a rather favorable myelotoxic profile, with a relatively low incidence of grade 3-4 events $(<10 \%[3,4])$. However, TMZ is a relatively new agent and the prognosis of patients treated with TMZ is usually short due to the underlying malignancy. Consequently, severe hematological adverse events may not have been characterized yet [5].

In this paper, we present a patient who developed a high-grade B-cell lymphoma after being treated with an extended dose-dense schedule of TMZ. To our knowledge, there are only a few case reports of this type. This suggests that there might be an increased risk of non-Hodgkin lymphoma after an extended dose-dense TMZ treatment.

In their study of severe hematologic adverse events associated with TMZ reported between 1997 and 2008, Villano et al. [6] found reports of myelodysplastic syndromes, agranulocytosis, pancytopenia, leukemia (both acute lymphoblastic and acute myeloid), and lymphoma. They also found a (rather concerning) high rate of aplastic anemia, which appears to be specifically associated with TMZ. These authors conclude that TMZ presents a profile of hematologic toxicity different from that of other alkylating agents.

Remarkable in the present case was the development of a grade 3-4 lymphopenia early in treatment. Histological analysis indicated a high-grade B-cell lymphoma (diffuse large Bcell lymphoma, DLBCL). The global image, rather unusual for a 'sporadic' DLBCL, is more consistent with post-transplant lymphoproliferative disorders, as corroborated by the presence of CD30-positive cells.

Furthermore, the EBV genome was present in the proliferation. The precise contribution of EBV to the pathogenesis is unclear, but EBV can cause abnormal growth of infected B-cells and some tumors are indeed known to be EBV associated (e.g. Burkitt's lymphoma, Hodgkin disease, nasopharyngeal carcinoma, post-transplant lymphoma). However, considering the high incidence of EBV infection, the finding of it might be random.

The mechanism by which secondary (hematological) neoplasms occur after treatment with TMZ remains largely unknown, though it is most likely related to treatment dose and duration. It might be possible that defective DNA repair (due to depletion of MGMT) leads to genome instability and hypermutation, thereby predisposing leukemogenesis [3]. Alternatively, immunosuppressed patients (post-transplant, HIV-infected) are known to be at an increased risk of developing lymphoproliferative disorders, most of these being B-cell derived and EBV associated [7]. 
Primary infection by EBV causes a proliferation of EBV-infected B-cells. This elicits a cytotoxic T-lymphocyte response which allows the transition into asymptomatic life-long infection. The persisting lymphopenia and thus the breakdown of EBV-specific immunity, may have contributed to the development of EBV-associated malignancy [7]. There are also cases of high-grade B-cell lymphoma occurring at the site of a chronic inflammatory process (pyothorax or osteomyelitis), where chronic inflammation results in a local defect of the EBV-specific immunity [7]. Thus, both the immunosuppressive and the possible mutagenic effect of TMZ can explain why our patient developed non-Hodgkin B-cell lymphoma. Further investigation is warranted to disentangle the influence of these mechanisms.

Establishing a (unequivocal) causal relationship between TMZ administration and secondary malignancies is difficult, since many patients receive additional therapies, including different alkylating agents and radiation therapy. Nevertheless, this report of a patient developing non-Hodgkin B-cell lymphoma after TMZ treatment highlights the importance of regular (hematological) follow-up of patients and emphasizes the need of reporting major adverse events associated with TMZ. As the use of TMZ against various neoplasms associated with a better prognosis (hence, a longer survival) increases, the awareness that TMZ may induce secondary malignancies through its cytotoxic and mutagenic properties should increase as well. Based on this report, we would recommend a higher degree of vigilance in patients who develop grade 3-4 lymphopenia.

\section{Disclosure Statement}

There were no conflicts of interest.

\section{Acknowledgements}

I would like to express my very great appreciation to Dr. Jeroen Mebis, who made it possible for me to write this case report, and I am particularly grateful for the assistance given by Sandrine Censabella, $\mathrm{PhD}$, in editing this paper.

\section{References}

1 Sharma A, Gupta D, Mohanti BK, Thulkar S, Dwary A, Goyal S, Muzumder S, Das P: Non-Hodgkin lymphoma following temozolomide. Pediatr Blood Cancer 2009;53:661-662.

-2 Neyns B, Cordera S, Joosens E, Nader P: Non-Hodgkin's lymphoma in patients with glioma treated with temozolomide. J Clin Oncol 2008;26:4518-4519.

-3 Momota H, Nariata Y, Miyakita Y, Hosono A, Makimoto A, Shibui S: Acute lymphoblastic leukemia after temozolomide treatment for anaplastic astrocytoma in a child with a germline TP53 mutation. Pediatr Blood Cancer 2010;55:577-579.

-4 Su YW, Chang MC, Chiang MF, Hsieh RK: Treatment-related myelodysplastic syndrome after temozolomide for recurrent high-grade glioma. J Neurooncol 2005;71:315-318.

5 Ogura M, Todo T, Tanaka M, Nannya Y, Ichikawa M, Nakamura F, Kurokawa M: Temozolomide may induce therapy-related acute lymphoblastic leukaemia. Br J Haematol 2011;154:663-665.

-6 Villano J, Letarte N, Yu J, Abdur S, Bressler L: Hematologic adverse events associated with temozolomide. Cancer Chemother Pharmacol 2012;69:107-113.

7 Niedobitek G, Meru N, Delecluse HJ: Epstein-Barr virus infection and human malignancies. Int J Exp Pathol 2001;82:149-170. 
Van Ginderachter et al.: Non-Hodgkin Lymphoma after Treatment with Extended Dosing Temozolomide and Radiotherapy for a Glioblastoma: A Case Report

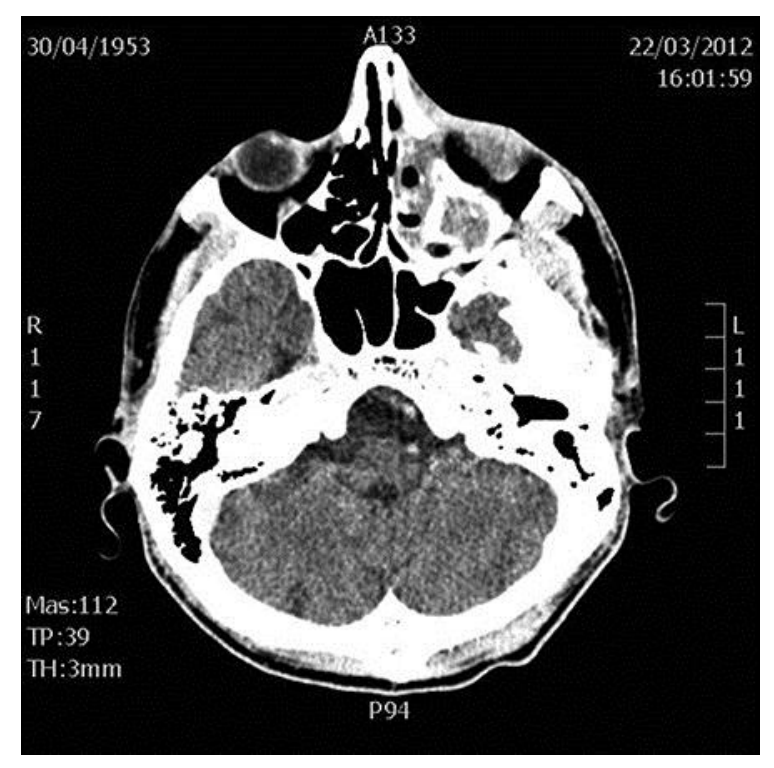

Fig. 1. CT scan.

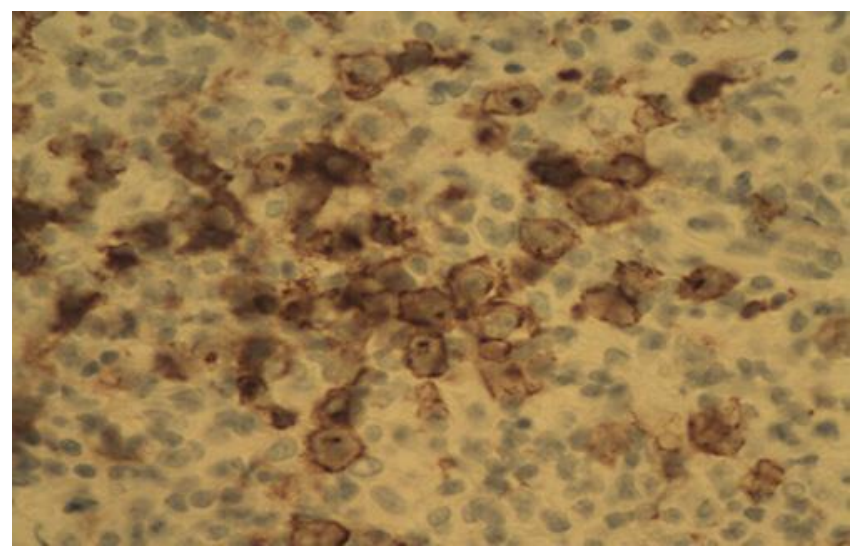

Fig. 2. Microscopic view: CD30-positive cells. 